\title{
Avaliação do pH ácido e potencial erosivo de bebidas alcoólicas Evaluation of acid $\mathrm{pH}$ and erosive potential of alcoholic beverages Evaluación del pH ácido y potencial erosivo de bebidas alcohólicas Rainier Almeida de MEDEIROS ${ }^{1}$ José de Alencar FERNANDES NETO Maria Helena Chaves de Vasconcelos CATÃo ${ }^{3}$ \\ ${ }^{1}$ Cirurgião-Dentista, Departamento de Odontologia, Universidade Estadual da Paraíba - UEPB 58429-500, Campina Grande - PB, Brasil \\ ${ }^{2}$ Doutorando, Programa de Pós-Graduação em Odontologia, Departamento de Odontologia, Universidade Estadual da Paraíba - UEPB 58429-500, Campina Grande - PB, Brasil \\ ${ }^{3}$ Professora Doutora do Programa de Pós-Graduação em Odontologia, Universidade Estadual da Paraíba - UEPB 58429-500, Campina Grande - PB, Brasil
}

\begin{abstract}
Resumo
Introdução: A erosão dental é definida como um processo patológico que leva à perda de substância dentária por processos químicos. Atualmente, os fatores de risco para a ocorrência dessa lesão têm sido pesquisados com maior interesse, já que sua incidência aumenta, devido às mudanças nos hábitos alimentares, desordens gastrointestinais e descontrole alimentar psicológico. Objetivo: Avaliar o pH de quatro tipos de bebidas alcoólicas (Smirnoff Ice ${ }^{\circledR}$, Talante ${ }^{\circledR}$ sabor coco, canela e menta) em duas temperaturas $\left(25^{\circ} \mathrm{C}\right.$ e $5^{\circ} \mathrm{C}$ ), sob o ponto de vista do potencial erosivo. Material e método: Foram avaliados oito exemplares de cada bebida nas temperaturas determinadas com o auxílio do aparelho pHmetro. Resultados: Todas as amostras das bebidas avaliadas apresentaram $\mathrm{pH}$ inferior a 5,0. O pH da Smirnoff Ice ${ }^{\circledR}$ é significativamente menor do que qualquer que seja o sabor da Talante ${ }^{\circledR}$. A Smirnoff Ice ${ }^{\circledR}$ apresentou a média do $\mathrm{pH} 2,34$, sendo significativamente menor do que as demais bebidas. Quanto à temperatura os resultados mostraram que a mesma não altera o pH da bebida Talante ${ }^{\circledR}$ independente do sabor. Conclusão: Existem evidências de que a temperatura altera significativamente o $\mathrm{pH}$ da bebida Smirnoff Ice ${ }^{\circledR}$. Se consumidas com frequência, as bebidas avaliadas podem contribuir para o desenvolvimento de erosão dentária.

Descritores: Erosão Dentária; Bebidas Alcoólicas; Propriedades Físicas e Químicas; Concentração de Íons de Hidrogênio.
\end{abstract}

\section{Abstract}

Introduction: Dental erosion is defined as a pathological process that leads to the loss of dental substance by chemical processes. Currently, the risk factors for the occurrence of this lesion have been investigated with greater interest, since its incidence increases due to changes in eating habits, gastrointestinal disorders and psychological eating disorder. Objective: To evaluate the $\mathrm{pH}$ of four types of alcoholic beverages (Smirnoff Ice ${ }^{\mathrm{TM}}$, Talante ${ }^{\mathrm{TM}}$ coconut flavor, Talante $^{\mathrm{TM}}$ cinnamon flavor, Talante $^{\mathrm{TM}}$ mint flavor) at two temperatures $\left(25^{\circ} \mathrm{C}\right.$ and $5^{\circ} \mathrm{C}$ ), in the view of erosive potential. Material and methods: There were eight copies of each drink in temperatures determined with the aid of a pHmeter. Results: All samples of drinks evaluated presented $\mathrm{pH}$ less than 5.0. The $\mathrm{pH}$ of Smirnoff Ice ${ }^{\mathrm{TM}}$ is significantly lower than whatever the flavor of Talante ${ }^{\mathrm{TM}}$. The Smirnoff Ice ${ }^{\mathrm{TM}}$ had an average $\mathrm{pH}$ of 2.34 , significantly less than other drinks. As for the temperature the results show that it doesn't change the $\mathrm{pH}^{\mathrm{T}}$ of the Talante ${ }^{\mathrm{TM}}$ beverage independent of flavor. Conclusion: However, there is evidence that the temperature alters significantly the pH of the drink Smirnoff Ice $^{\mathrm{TM}}$. If frequently consumed, the beverages evaluated can contribute to the development of dental erosion.

Descriptors: Tooth Erosion; Alcoholic Beverages; Physical and Chemical Properties; Hydrogen-Ion Concentration.

\section{Resumen}

Introducción: La erosión dental se define como un proceso patológico que lleva a la pérdida de sustancia dental por procesos químicos. En la actualidad, los factores de riesgo para la ocurrencia de esta lesión han sido investigados con mayor interés, ya que su incidencia aumenta, debido a los cambios en los hábitos alimentarios, desórdenes gastrointestinales y descontrol dietético. Objetivo: Evaluar el pH de cuatro tipos de bebidas alcohólicas (Smirnoff Ice ${ }^{\circledR}$, Talante ${ }^{\circledR}$ sabor coco, canela y menta) en dos temperaturas $\left(25^{\circ} \mathrm{C}\right.$ y $5^{\circ} \mathrm{C}$ ), desde el punto de vista del potencial erosivo. Material y método: Se evaluaron ocho ejemplares de cada bebida en las temperaturas determinadas con la ayuda del aparato pHmetro. Resultados: Todas las muestras de las bebidas evaluadas presentaron un pH inferior a 5,0. El pH de la Smirnoff Ice ${ }^{\circledR}$ es significativamente menor que cualquiera que sea el sabor de la Talante ${ }^{\circledR}$. La Smirnoff Ice ${ }^{\circledR}$ presentó la media del pH 2,34, siendo significativamente menor que las demás bebidas. En cuanto a la temperatura los resultados mostraron que la misma no altera el pH de la bebida Talante ${ }^{\circledR}$ independiente del sabor. Conclusión: Existen evidencias de que la temperatura altera significativamente el $\mathrm{pH}$ de la bebida Smirnoff Ice ${ }^{\circledR}$. Si se consumen con frecuencia, las bebidas evaluadas pueden contribuir al desarrollo de la erosión dental.

Descriptores: Erosión de los Dientes; Bebidas Alcohólicas; Propiedades Físicas y Químicas; Concentración de Iones de Hidrógenio.

\section{INTRODUÇÃO}

A erosão dental é definida como uma entidade patológica, que leva à perda de substância dentária por processos químicos que não envolvem microrganismos. Pode ser classificada de acordo com a sua etiologia como extrínseca (causada por ácidos exógenos), intrínseca (ácidos endógenos) ou idiopática (ácidos de origem desconhecida) $)^{1,2}$.

Os fatores extrínsecos são a principal causa de erosão constatada. Dentre eles, a dieta é o de maior importância no que diz respeito à perda de estrutura dental. Há uma maior preocupação principalmente entre indivíduos jovens, que tendem a ter uma dieta rica em alimentos e bebidas ácidas, como o grande consumo de refrigerantes, sucos artificiais e naturais, além da elevada ingestão de bebidas alcoólicas ${ }^{3}$.

Nas duas últimas décadas, os fatores de risco para a ocorrência dessa lesão têm sido pesquisados com maior interesse. Em países desenvolvidos, a incidência de cárie diminuiu, enquanto que o risco de ocorrerem lesões de erosão aumentou, como resultado de mudanças nos hábitos alimentares, desordens gastrointestinais e descontrole alimentar psicológico ${ }^{4}$.
A prevenção e controle da erosão podem ser efetuados por meio da diminuição da frequência de ingestão ou da redução do potencial erosivo de bebidas ácidas ${ }^{5}$. De fato, já foi demonstrado que sua erosividade ocorre na dependência de fatores químicos, tais como tipo de ácido, $\mathrm{pH}$, acidez titulável, potencial quelante, concentração de cálcio, de fosfato e de flúor, e de aspectos físicos, como temperatura ${ }^{6,7} \mathrm{e}$ adesividade $^{8}$

Desse modo, apesar da fisiopatologia da erosão dental estar relacionada também a fatores biológicos e comportamentais, o entendimento do papel dos aspectos físico-químicos relacionados ao caráter erosivo de bebidas contribui para fundamentar estratégias que visem minimizar a formação e a progressão das lesões de erosão. Embora, isoladamente, o principal indicador do potencial erosivo de bebidas seja o $\mathrm{pH}$, as demais propriedades físico-químicas podem afetar significativamente sua capacidade erosiva ${ }^{9,10}$.

Potencial hidrogeniônico $(\mathrm{pH})$ é uma grandeza físicoquímica que permite indicar se um meio aquoso é ácido (valor inferior a 7) ou básico (valor superior a 7). A mensuração do 
$\mathrm{pH}$, isoladamente, representa o melhor parâmetro para avaliação do potencial erosivo de bebidas ácidas ${ }^{10} . \mathrm{O} \mathrm{pH}$, juntamente com seu conteúdo de cálcio, fosfato e flúor, determina o grau de saturação de uma bebida em relação à estrutura dental e, consequentemente, seu poder de dissolução. Assim, soluções supersaturadas em relação ao esmalte e à dentina não promovem dissolução mineral, ao passo que as subsaturadas proporcionam a perda mineral desses tecidos ${ }^{11}$.

O fenômeno da erosão dentária vem sendo tema constante de relatos de casos clínicos e pesquisas. O maior tempo de permanência dos elementos dentários na cavidade bucal, como consequência direta do desenvolvimento de medidas preventivas para o controle da cárie, associado a mudanças no estilo de vida, principalmente no que se refere a hábitos alimentares, fez com que a classe odontológica atentasse para outros problemas; dentre estes, a erosão ${ }^{12}$.

As bebidas ácidas aumentam a permeabilidade dentinária pela remoção e dissolução da smear layer, assim como pela desmineralização da matriz intertubular que promove a ampliação dos túbulos dentinários levando a um aumento na permeabilidade ${ }^{13}$. A grande oferta de bebidas no mercado, o fácil acesso, o baixo custo e a diversidade fazem questionar a possibilidade de algumas delas estarem relacionadas ao desenvolvimento de lesões por erosão ${ }^{14}$.

Sabendo-se que a erosão dental é um processo patológico crescente atualmente, e que a mesma é causada principalmente por fatores extrínsecos, este estudo teve a finalidade de avaliar o $\mathrm{pH}$ ácido e o potencial erosivo em bebidas alcoólicas acessíveis no mercado consumidor.

\section{MATERIAL E MÉTODO}

O estudo caracterizou-se como sendo experimental in vitro com observação direta em laboratório, destinado a avaliar o pH de bebidas alcoólicas acessíveis no mercado. Foram utilizados quatro tipos de bebidas alcoólicas tradicionais na região: Smirnoff Ice ${ }^{\circledR}$; Cachaça Talante ${ }^{\circledR}$ sabor coco, menta e canela, adquiridas em estabelecimentos comerciais do município de Campina Grande na Paraíba (Quadro 1).

Os seguintes critérios foram utilizados: as bebidas deveriam estar fechadas, lacradas e dentro do prazo de validade e aquelas cujo lacre possuía alguma falha, trincas na embalagem ou qualquer fator que pudesse vir modificar o $\mathrm{pH}$, bem como os prazos de validade vencidos não foram incluídas na amostra.

Com a finalidade de conhecer o $\mathrm{pH}$ e o potencial erosivo de algumas bebidas alcoólicas industrializadas bastante consumidas na região do Nordeste, foram selecionados 8 exemplares de cada bebida (Smirnoff Ice ${ }^{\circledR}$; Cachaça Talante ${ }^{\circledR}$ coco, menta e canela), sendo as mesmas avaliadas resfriadas (temperatura de $\pm 5^{\circ} \mathrm{C}$ ) e a temperatura ambiente $\left( \pm 25^{\circ} \mathrm{C}\right)$; perfazendo um total de 62 amostras avaliadas.

$\mathrm{O}$ aparelho utilizado para as medições do $\mathrm{pH}$ endógeno foi um pHmetro (Micronal) com eletrodos de vidro e mostrador digital (HI 221 Calibration Check Microprocessor pH Meter - HANNA instruments) do Centro de Ciências Tecnológicas da Universidade Estadual da Paraíba do Departamento de Química.

O método utilizado foi o potenciométrico. Após a calibração do aparelho com soluções padronizadas com valores de $\mathrm{pH}=7,0$ e $\mathrm{pH}=4,0$ ajustou-se a temperatura das bebidas ao aparelho.

As bebidas foram avaliadas logo após a retirada do lacre e medidas três vezes consecutivas. Imediatamente após a abertura das bebidas naturais, transferiu-se com uma pipeta $50 \mathrm{ml}$ de cada bebida para um becker estéril e aferiu-se os valores de $\mathrm{pH}$, sendo realizadas três medidas consecutivas do $\mathrm{pH}$ de cada bebida, seguida da leitura e do registro em ficha específica e obtendo a média na temperatura ambiente.

Em seguida, as bebidas foram colocadas para gelar até atingirem uma temperatura aproximada de $\pm 5^{\circ} \mathrm{C}$, realizandose novamente a mensuração do $\mathrm{pH}$, seguindo as mesmas etapas supracitadas para as bebidas em temperatura ambiente $\left( \pm 25^{\circ} \mathrm{C}\right)$.

Trinta minutos antes da medição do $\mathrm{pH}$, o potenciômetro foi ligado em $S T A N D-B Y$, realizando-se o ajuste com o auxílio do controle SET.STD1 no display para 7,10. O eletrodo de medição foi imergido na solução tampão $\mathrm{pH}=7,0$. Depois de 15 segundos, colocou-se o controle STAND-BY/MEAS na posição $M E A S$ e o valor foi ajustado no display para o pH 7,0 com o auxílio do controle STD1. Colocou-se o controle STAND-BY/MEAS na posição STAND$B Y$, retirou-se o eletrodo da solução tampão, lavou-o com água destilada e secou-o com papel absorvente. Esse mesmo procedimento executado com a solução de $\mathrm{pH}=7,0$, foi realizado também com a solução tampão $\mathrm{pH}=4,0$. O ajuste foi repetido até que o pHmetro atingisse a reprodutibilidade adequada. Ao atingir, imergiu-se o eletrodo de medição na amostra de $50 \mathrm{ml}$, aguardou-se 15 segundos, colocou-se o controle STAND-BY/MEAS na posição $M E A S$ e o valor foi mostrado no display. Entre cada medição diferente, o eletrodo foi lavado com água destilada e secado com papel absorvente.

Os dados foram digitados e analisados no SPSS (Statistical Package for Social Sciences) na versão 13.0. A margem de erro dos testes estatísticos foi de 5\%. Depois foram analisados através de técnicas estatísticas descritivas e inferenciais sendo as primeiras, por meio de gráficos e tabela, enquanto a análise inferencial foi realizada por meio de comparação de médias e comparações múltiplas. Os testes utilizados foram $t$ de Student para amostras independentes, $F$ de Sneedecor (ANOVA), teste de Tukey, teste de Levene e Shapiro-Wilk.

Quadro 1. Nome comercial, tipo, sabor e fabricante dos produtos investigados

\begin{tabular}{|c|c|c|c|}
\hline Nome comercial & Tipo de produto & Sabor & Fabricante \\
\hline Smirnoff Ice $^{\circledR}$ & Vodka & Limão & Diageo Brasil \\
\hline Talante $^{(}$ & Cachaça & Coco & Talantemix \\
\hline Talante $^{\circledR}$ & Cachaça & Menta & Talantemix \\
\hline Talante $^{(}$ & Cachaça & Canela & Talantemix \\
\hline
\end{tabular}

\section{RESULTADOS}

Verificou-se que a bebida Talante ${ }^{\circledR}$ sabor coco, $\mathrm{pH}=4,17$, apresentou o maior $\mathrm{pH}$ entre as bebidas em estudo, enquanto que a Smirnoff Ice ${ }^{\circledR}$ apresentou o menor pH médio, 2,34 (Tabela 1). Por meio da análise de variância, teste $\mathrm{F}$ de Sneedecor $(p<0,01)$ constatou-se evidências estatísticas de que o $\mathrm{pH}$ diferiu significativamente entre as bebidas, ao nível de $5 \%$ de significância.

Tabela 1. Distribuição dos produtos investigados segundo o $\mathrm{pH}$ médio e desvio padrão

\begin{tabular}{lcccc}
\hline Bebidas & $\begin{array}{c}\text { pH } \\
\text { médio }\end{array}$ & n & Desvio padrão & P valor \\
\hline Smirnoff Ice $^{(B)}$ & 2,34 & 8 & 0,080 & \\
Talante sabor coco $^{(B)}$ & 4,17 & 8 & 0,029 & \\
Talante sabor menta $^{(B)}$ & 3,57 & 8 & 0,029 & $<0,01$ \\
Talante sabor canela $^{(B)}$ & 3,48 & 8 & 0,031 & \\
\hline Total & $\mathbf{3 , 3 9}$ & $\mathbf{3 2}$ & $\mathbf{0 , 6 7 4}$ & \\
\hline
\end{tabular}

Verificou-se, através do teste de Tukey ao nível de $5 \%$ de significância, que o pH da Smirnoff Ice ${ }^{\circledR}$ foi significativamente menor do o $\mathrm{pH}$ de qualquer que fosse $\mathrm{o}$ sabor da Talante ${ }^{\circledR}$. O pH da Talante sabor coco, 4,17 foi significativamente maior do que os pHs da Smirnoff Ice ${ }^{\circledR}$, da Talante ${ }^{\circledR}$ menta e Talante ${ }^{\circledR}$ canela. $\mathrm{O}$ pH da Talante ${ }^{\circledR}$ sabor menta, 3,57 foi significativamente maior que $\mathrm{o} \mathrm{pH}$ da Smirnoff Ice ${ }^{\circledR}$ e Talante $^{\circledR}$ sabor canela, revelando-se inferior apenas ao $\mathrm{pH}$ da Talante ${ }^{\circledR}$ coco. $\mathrm{O}$ pH da Talante ${ }^{\circledR}$ sabor 
canela, 3,48, é significativamente maior do que o $\mathrm{pH}$ da Smirnoff Ice ${ }^{\circledR}$, porém, menor significativamente do que o $\mathrm{pH}$ da Talante ${ }^{\circledR}$ coco e da Talante ${ }^{\circledR}$ sabor menta (Tabela 2).

Por meio do teste $t$ de Student $\mathrm{p}=0,008$, foi constatado que o pH médio 2,402 da bebida Smirnoff Ice $^{\circledR}$ em temperatura ambiente, aproximadamente $27^{\circ} \mathrm{C}$ foi significativamente maior do que o $\mathrm{pH}, 2,275$ em temperatura de aproximadamente $5^{\circ} \mathrm{C}$ ao nível de $5 \%$ de significância. A pesquisa evidenciou que o $\mathrm{pH}$ da bebida Talante ${ }^{\circledR}$ sabor coco, pH médio de 4,167 não diferenciou significativamente entre as variações de temperatura $\left( \pm 5^{\circ} \mathrm{C} \mathrm{e} \pm 25^{\circ} \mathrm{C}\right)(\mathrm{p}=1,000)$.

$\mathrm{O}$ pH das bebidas Talante ${ }^{\circledR}$ sabor menta e Talante ${ }^{\circledR}$ sabor canela foram diferentes entre si sendo eles 3,572 e 3,477 , respectivamente. Porém, pelo teste $\mathrm{t}$ de Student $\mathrm{p}=1,000$, não se verificou diferença estatística significativa nos $\mathrm{pHs}$ entre os dois grupos (temperatura ambiente $\mathrm{e}$ temperatura resfriada) $(\mathrm{p}=1,000)$.

Foram encontradas evidências de que a temperatura não altera o $\mathrm{pH}$ da bebida Talante ${ }^{\circledR}$ independente do sabor. Todavia, altera significativamente o $\mathrm{pH}$ da bebida Smirnoff Ice $^{\circledR}$. A Tabela 3 mostra os valores do $\mathrm{pH}$ das diferentes bebidas nas temperaturas ambiente e resfriada.

Tabela 2. Distribuição das bebidas na análise de comparações múltiplas dos pHs entre as bebidas.

\begin{tabular}{|c|c|c|c|c|c|}
\hline \multirow{2}{*}{$\begin{array}{c}\text { (I) } \\
\text { Bebida }\end{array}$} & \multirow{2}{*}{$\begin{array}{c}(\mathbf{J}) \\
\text { Bebida }\end{array}$} & \multirow{2}{*}{$\begin{array}{c}\text { Diferença } \\
\text { média } \\
\text { (I-J) }\end{array}$} & \multirow[b]{2}{*}{$\begin{array}{c}\mathbf{P} \\
\text { valor }\end{array}$} & \multicolumn{2}{|c|}{$\begin{array}{c}\text { Intervalo de } \\
\text { confiança com } 95 \% \\
\text { de confiança }\end{array}$} \\
\hline & & & & $\begin{array}{c}\text { Limite } \\
\text { inferior }\end{array}$ & $\begin{array}{c}\text { Limite } \\
\text { Superior }\end{array}$ \\
\hline \multirow{3}{*}{$\underset{\text { Ice }^{\circledR}}{\text { Smirnoff }}$} & Talante $^{\circledR}$ sabor coco & $-1,829^{*}$ & 0,00 & $-1,894$ & $-1,764$ \\
\hline & Talante $^{\circledR}$ sabor menta & $-1,234^{*}$ & 0,00 & $-1,299$ & $-1,169$ \\
\hline & Talante $^{\circledR}$ sabor canela & $-1,139^{*}$ & 0,00 & $-1,204$ & $-1,074$ \\
\hline \multirow{3}{*}{$\begin{array}{c}\text { Talante }^{\circledR} \\
\text { sabor coco }\end{array}$} & Smirnoff Ice ${ }^{(B)}$ & $1,829^{*}$ & 0,00 & 1,764 & 1,894 \\
\hline & Talante $^{\circledR}$ sabor menta & $0,595^{*}$ & 0,00 & 0,530 & 0,659 \\
\hline & Talante $^{\circledR}$ sabor canela & $0,690^{*}$ & 0,00 & 0,625 & 0,755 \\
\hline \multirow{3}{*}{$\begin{array}{c}\text { Talante }^{\circledR} \\
\text { sabor menta }\end{array}$} & Smirnoff Ice ${ }^{\circledR}$ & $1,234^{*}$ & 0,00 & 1,169 & 1,299 \\
\hline & Talante ${ }^{(\otimes)}$ sabor coco & $-0,595^{*}$ & 0,00 & $-0,659$ & $-0,530$ \\
\hline & Talante $^{(\otimes)}$ sabor canela & $0,095^{*}$ & 0,002 & 0,030 & 0,160 \\
\hline \multirow{3}{*}{$\begin{array}{c}\text { Talante }^{\circledR} \\
\text { sabor canela }\end{array}$} & Smirnoff Ice ${ }^{\circledR}$ & $1,139^{*}$ & 0,00 & 1,074 & 1,204 \\
\hline & Talante ${ }^{\circledR}$ sabor coco & $-0,690^{*}$ & 0,00 & $-0,755$ & $-0,625$ \\
\hline & Talante $^{\circledR}$ sabor menta & $-0,095^{*}$ & 0,002 & $-0,160$ & $-0,030$ \\
\hline
\end{tabular}

Tabela 3. Distribuição das bebidas segundo o $\mathrm{pH}$ médio, desvio padrão em diferentes temperaturas

\begin{tabular}{|c|c|c|c|c|c|}
\hline Bebidas & Temperatura & $\begin{array}{c}\text { pH } \\
\text { Médio }\end{array}$ & $\mathbf{n}$ & $\begin{array}{l}\text { Desvio } \\
\text { padrão }\end{array}$ & $P$ valor \\
\hline \multirow{2}{*}{ Smirnoff Ice ${ }^{\circledR}$} & Temperatura ambiente & 2,402 & 4 & 0,04113 & \multirow{2}{*}{0,008} \\
\hline & Temperatura fria & 2,275 & 4 & 0,05000 & \\
\hline \multirow{4}{*}{$\begin{array}{c}\text { Talante }{ }^{\circledR} \text { sabor } \\
\text { coco }\end{array}$} & Total & 2,339 & 8 & 0,08026 & \multirow{4}{*}{1,000} \\
\hline & Temperatura ambiente & 4,167 & 4 & 0,03096 & \\
\hline & Temperatura fria & 4,167 & 4 & 0,03096 & \\
\hline & Total & 4,167 & 8 & 0,02866 & \\
\hline \multirow{2}{*}{$\begin{array}{c}\text { Talante }^{\circledR} \text { sabor } \\
\text { menta }\end{array}$} & $\begin{array}{l}\text { Temperatura } \\
\text { ambiente }\end{array}$ & 3,572 & 4 & 0,03096 & \multirow[t]{2}{*}{1,000} \\
\hline & Temperatura fria & 3,572 & 4 & 0,03096 & \\
\hline \multirow{4}{*}{$\begin{array}{c}\text { Talante }{ }^{\circledR} \text { sabor } \\
\text { canela }\end{array}$} & Total & 3,572 & 8 & 0,02866 & \multirow{4}{*}{1,000} \\
\hline & $\begin{array}{l}\text { Temperatura } \\
\text { ambiente }\end{array}$ & 3,477 & 4 & 0,03304 & \\
\hline & Temperatura fria & 3,477 & 4 & 0,03304 & \\
\hline & Total & 3,477 & 8 & 0,03059 & \\
\hline
\end{tabular}

\section{DISCUSSÃO}

A erosão dental é o resultado de uma perda patológica e crônica de tecidos duros do dente sem envolvimento bacteriano ${ }^{5}$. A erosão difere da cárie dentária, pois esta ocorre em áreas cobertas por biofilme, enquanto a erosão ocorre em áreas livres de biofilme, em todas as superfícies expostas ${ }^{2}$.

A etiologia da erosão está relacionada a ácidos que não são produzidos pela ação de bactérias cariogênicas, mas pela presença regular de substâncias ácidas em pH normalmente inferior ao $\mathrm{pH}$ em que o flúor consegue ativar o processo de remineralização, ou seja, menor que $4,5^{15}$. Entretanto, bebidas que possuem $\mathrm{pH}$ inferior a 5,5 já podem causar erosão, principalmente se a exposição dos minerais dentários for frequente e de longa duração ${ }^{16}$. O entendimento do papel dos aspectos físico-químicos relacionados ao caráter erosivo de bebidas ácidas contribui para fundamentar estratégias que visam minimizar a formação e a progressão de lesões de erosão ${ }^{17}$. Vários estudos mencionam as bebidas alcoólicas como agentes que possuem baixo $\mathrm{pH}$ baixo ${ }^{18-21}$.

No presente estudo, o $\mathrm{pH}$ médio de 2,34 da Smirnoff Ice $^{\circledR}$ foi significativamente menor do que qualquer que seja o sabor da Talante ${ }^{\circledR}$, apresentando, portanto, um pH ácido passível de desmineralizar o esmalte, caso seja consumido diariamente e em excesso. Entretanto a presença de dados na literatura que identificam os valores do $\mathrm{pH}$ bem como o potencial erosivo dessas bebidas são escassos.

Diante dos achados do presente trabalho e da literatura consultada, e considerando-se que a ingestão de bebidas ácidas se mostra relacionada ao desenvolvimento de lesões de erosão dental, a necessidade de um melhor esclarecimento aos consumidores sobre as características dessas bebidas, em especial acerca do $\mathrm{pH}$ ácido das mesmas, torna-se necessário.

Dessa forma, optou-se por medir o $\mathrm{pH}$ de bebidas alcoólicas bastante consumidas na região, objetivando com isso oferecer um melhor embasamento na orientação dos pacientes quanto ao consumo das mesmas, o que é fundamental durante o tratamento das lesões de erosão dental.

Foram analisadas 32 amostras, sendo 24 da bebida Talante ${ }^{\circledR}$ de sabores diferentes, coco, menta e canela, e 8 da bebida Smirnoff Ice $^{\circledR}$. Em cada bebida, foi verificado e comparado seu $\mathrm{pH}$ na temperatura ambiente e fria. Além disso, foi empregado 8 exemplares de cada bebida para que fosse possível estabelecer uma simples comparação e com isso obter-se valores mais fidedignos do $\mathrm{pH}$ das mesmas, sendo esse dado extremamente útil no momento de se orientar sobre a influência do consumo dessas bebidas pelo paciente no tratamento e na prevenção das lesões de erosão dental. Foi observado que todas as bebidas avaliadas neste estudo apresentaram um $\mathrm{pH}$ abaixo de 4,5. Além disso, uma delas, a Smirnoff Ice ${ }^{\circledR}$, apresentou o pH abaixo de 3,0. Verificou-se ainda que o $\mathrm{pH}$ da bebida Smirnoff Ice ${ }^{\circledR}$ em temperatura ambiente é maior do que na temperatura de $5^{\circ} \mathrm{C}$. Esses achados sugerem que o consumo dessas bebidas se constitui um fator de risco para o desenvolvimento da erosão nas estruturas dentárias ${ }^{15,16}$.

As bebidas do tipo ice com vodka também têm sido relacionadas à formação de lesões de erosão ${ }^{22}$ e no presente estudo foi a que apresentou o menor $\mathrm{pH}$. Entretanto, apesar da importância da mensuração do $\mathrm{pH}$ na avaliação do aparecimento de lesões erosivas, somente o $\mathrm{pH}$ de uma substância da dieta não é capaz de avaliar o seu potencial erosivo. A acidez titulável é considerada mais importante do que o $\mathrm{pH}$, e a propriedade de quelação de cálcio pode intensificar o potencial erosivo de substâncias da dieta ${ }^{23}$.

Os resultados da literatura avaliada justificam a preocupação que motivaram a realização deste estudo, uma vez que, o consumo das bebidas avaliadas é frequente entre os jovens e adolescentes e, até o momento, existem poucas informações sobre o seu efeito na superfície do esmalte dentário e em materiais restauradores utilizados na odontologia. Nesse caso, faz-se necessário a realização de trabalhos adicionais que comprovem os achados desse estudo, bem como que pesquisem sobre os demais fatores associados ao potencial erosivo nessas bebidas.

\section{CONCLUSÃO}

De acordo com os resultados obtidos foi possível concluir que todas as bebidas (Smirnoff Ice ${ }^{\circledR}$, Talante ${ }^{\circledR}$ sabor 
coco, Talante ${ }^{\circledR}$ sabor canela e Talante $^{\circledR}$ sabor menta) apresentam um pH abaixo de 4,5. O pH da Smirnoff ice ${ }^{\circledR}$ é significativamente menor do que o $\mathrm{pH}$ das outras bebidas avaliadas, sugerindo seu maior potencial erosivo. A temperatura não altera o pH da bebida Talante ${ }^{\circledR}$, independente do sabor, entretanto, o pH da bebida Smirnoff Ice ${ }^{\circledR}$ altera-se significativamente com a temperatura. Se consumidas com frequência, as bebidas avaliadas podem contribuir para 0 desenvolvimento de erosão dentária.

\section{REFERÊNCIAS}

1. Zero DT, Lussi A. Erosion - chemical and biological factors of importance to the dental practitioner. Int Dent J. 2005; 55(Suppl 1):285-90.

2. Imfeld T. Dental erosion. Definition, classification and links. Eur J Oral Sci. 1996; 104(2):151-5.

3. Zero DT. Etiology of dental erosion - extrinsic factors. Eur J Oral Sci. 1996; 104(2):162-77.

4. Järvinen VK, Rytömaa II, Heinonen OP. Risk factors in dental erosion. J Dent Res. 1991; 70(6):942-47.

5. Lussi A, Jaeggi T, Schaffner M. Diet and dental erosion. Nutrition. 2002; 18(9):780-1.

6. Amaechi BT, Higham SM, Edgar WM. Factors influencing the development of dental erosion "in vitro" enamel type, temperature and exposure time. J Oral Rehabil. 1999, 26(8):624-30.

7. Barbour ME, Finke M, Parker DM, Hughes JA, Allen GC, Addy M. The relationship between enamel softening and erosion caused by soft drinks at a range of temperatures. J Dent. 2006; 34(3):207-13.

8. Ireland AJ, McGuinness $\mathrm{N}$, Sherriff $\mathrm{M}$. An investigation into the ability of soft drinks to adhere to enamel. Caries Res. 1995; 29(6):470-76.

9. Featherstone JD, Lussi A. Understanding the chemistry of dental erosion. Monogr Oral Sci. 2006; 20:66-76.

10. Hara AT, Zero DT. Analysis of the erosive potential of calcium-containing acidic beverages. Eur J Oral Sci. 2008; 116(1):60-5.

11. Lussi A, Jaeggi T. Chemical factors. Monogr Oral Sci. 2006; 20:77-87.

12. Amaechi BT, Higham SM. Dental erosion: possible approaches to prevention and control. J Dent. 2005; 33(3):243-52.

13. Mjör IA. Dentin-predentin complex and its permeability: pathology and treatment overview. J Dent Res. 1985; 64(Spec No):621-7.

14. Sobral MAP, Luz MAAC, Gama-Teixeira A, Garone Netto N. Influência da dieta líquida ácida no desenvolvimento de erosão dental. Pesqui Odontol Bras. 2000; 14(4):406-10.

15. Gandara BK, Truelove EL. Diagnosis and management of dental erosion. J Contemp Dent Pract. 1999; 15(1):16-23.

16. Corso AC, Hugo FN, Padilha DM. PH e tiratibilidade de sucos artificiais de limão. Rev Fac Odontol Porto Alegre. 2002; 43(1):30-3.

17. Furtado JR, Freire VC, Messias DC, Turssi CP. Aspectos físico-químicos relacionados ao potencial erosivo de bebidas ácidas. RFO. 2010; 15(3):323-8.

18. Smith BG, Robb ND. Dental erosion in patients with chronic alcoholism. J Dent. 1989; 17(5):219-21.

19. Grenby TH. Methods of assessing erosion and erosive potential. Eur J Oral Sci. 1996; 104(2 Pt2):207-14.

20. Matumoto MSS. Avaliação in vitro das alterações superficiais do esmalte dentário de dentes permanentes submetidos à consumo de bebidas energéticas [Tese de Doutorado]. São Paulo: Faculdade de Odontologia da Universidade de São Paulo; 2008.
21. Saiani RAS, Queiroz AM, Raffaini MSGG, BagatinRossi CR. Odontohebiatria: uma nova especialidade na odontologia. Rev Odontol Univ São Paulo. 2008; 20(1):60-5.

22. Rees J, Loyn T, Hunter L, Sadaghiani L, Gilmour A. The erosive potential of some flavored waters. Eur $\mathbf{J}$ Dent. 2007; 1(1):5-9.

23. West NX, Hughes JA, Addy M. The effect of $\mathrm{pH}$ on the erosion of dentine and enamel by dietary acids "in vitro". J Oral Rehabil. 2001; 28(9):860-64.

\section{CONFLITO DE INTERESSES}

Os autores declaram não haver conflitos de interesse.

\section{AUTOR PARA CORRESPONDÊNCIA}

Maria Helena Chaves de Vasconcelos Catão

mhelenact@zipmail.com.br

Submetido em 28/05/2018 Aceito em 30/06/2018 